\title{
Advanced Artificial Intelligence Assisted Learning Model for Smart Educational Learning Strategies for Teachers and Students
}

Xiaobing Yu ( $\sim$ bingruyu1@163.com )

Hunan Vocational college of Engineering

\section{Research Article}

Keywords: learning strategies, artificial intelligent technology, f-test, t-test, smart learning program

Posted Date: July 27th, 2021

DOI: https://doi.org/10.21203/rs.3.rs-739949/v1

License: (c) (i) This work is licensed under a Creative Commons Attribution 4.0 International License.

Read Full License 


\title{
Advanced Artificial Intelligence assisted Learning model for Smart educational learning strategies for teachers and students
}

\author{
$1^{*}$ XiaobingYu \\ ${ }^{1 *}$ Hunan Vocational College of Engineering, Changsha, Hunan, 410151,China \\ 1*Email: bingruyu1@163.com
}

\begin{abstract}
A smart classroom is a technology-based learning approach that helps to improve students ' skills in vocational colleges. It allows educators to create an interactive experience during teaching, which makes the system even more appealing and collaborative in learning strategies.In this paper, Advanced Artificial Intelligence assisted Learning model (AAILM)is used to design a smart interactive education system for vocational education.A qualitative approach is used on 40 vocational colleges around Chinato analyze thefindings, interviews, and focus group dialogue based on the learning strategies followed by students and teachers. Furthermore, the results of this analysis showed many improvements in the positions of teachers and students in the smart learning program.The study consisted of 102 students, and the results have been checkedbased on F-Test and T-Test. The result findings revealed that the academic successes of students in traditional and vocational colleges vary significantly and computed with graphical illustration as well as case study. The use of smart instruction has dramatically increased the success of teachers and students has been firmly researched in this paper. The simulation results illustrate that the suggestedmodelhas a high student's performance ratio of $98.6 \%$, a prediction ratio of $95.7 \%$, a student engagement ratio of $94.6 \%$, a task analysis ratio of $96.8 \%$, an efficiency ratio of $97.8 \%$ when compared to other existing methods.
\end{abstract}

Keywords: learning strategies, artificial intelligent technology, f-test, t-test, smart learning program

\section{Overview of Smart education in vocational education}

Smart learning involves different instructional environments in which the student and teacher focus on using technology[1]. Smart learningis structured in classrooms with online training in a combination ofsoftware and hardware available[2].The students are successful leaders rather than a static follow-up of the education process; they are made to digital information and skills more easily, quickly, and conveniently[3][4].In a smart classroom, digital 
whiteboards increasingly become indispensable learning with pictures is an open secret that allows students to understand complex topics even more[5].Smart teaching can be paired with the use of intelligent technology to improve conventional learning outcomes. Then an instructor can allow students to get more out of his/her curriculum knowledge using innovative teaching tools such as interactive online classes, a personalized learning platform, and the cloud platform[6][7].

In implementing AI technology and offering customized solutions to research abilities and needs in vocational colleges and it can boost their learning efficiency and teach without a teacher and examine the performance of students and analyse outcomes based on a huge volume of data[8]. Teachers who teach learning strategies for students to learn rather than teaching them a particular curriculum or skills[9]. The critical objective of learning techniques is to make students more successful[10]. The three most well-known learning methods are mnemonic, structural, and generative.Students take action to improve their learning methods[11]. Strategies are particularly crucial for verbal education because they are resources for active autonomous interaction central to enhancing communication skills.Starting class with a minute of attention is an essential cognitive ability, in which students have to become effective learners[12][13]. Students with varying learning strategies will enhance their concentration. It will enable students to become more conscious of their learning and to become independent through the creation of an increasing classroom [14].

Vocational education assists individuals with a great learning opportunity, with the improved results of their career. Jobs have the potential to develop their talents when earning profits.Technical and vocational education training (TVET) is essential for creating economic progress, the growth of employment, and the advancement of employment efficiency[15]. Technical and vocational education and preparation are significant in China. Vocational education and training (VET) for learners help in occupations that are manual or practical, typically non-universitarian, and entirely connected to a particular career, career, or vocation, hence the concept in which the apprentice is involved[16][17]. The improvement of domestic development and economic growth is essential for China to improve vocational education. Chinese companies are primarily faced with two concerns, low quality, and a lack of qualified labor [18]. These concerns impacted China's economic growth adversely and creativity, which results in soft quality goods, increased energy demand, and high industrial accident rates[19]. Interactive, insightful practices in the classroom forget one-way 
conversation or individual learning to connect and involve students with lessons and material [20].The involvement of smart boards allows teachers to give lectures more efficiently using numerous screens to illustrate the subject. Teachers can quickly demonstrate any aspect of the lesson[21].E-learning technology for vocational educationin china is based on a dynamic approach to knowledge sharing paper, pencil, and prints that are not required. They can transition into the 'Go Green Idea. Smart education is a way of approaching the formalized teaching-based education system is known as e-learning, utilizing electronic tools. Contrary to typical crab and instruction, e-Learning encourages and enhancesthe quality of learning. The use of computers and the Internet is the key aspect of E-learning, while lectures can be focused online or offline.

In this paper, Advanced Artificial Intelligence assisted Learning model (AAILM) has been proposed for a smart interactive education system for vocational education. Artificial intelligence supported e-learning platform are extensively utilized by vocational educational institutions to guarantee better teaching and learning experiences along withcareer enhancements.In addition, the convolutional neural network with Gated Recurrent Units has been introduced for prediction for students learning performance.In China, vocational education and training apply to train and professional qualifications offered by various programs such as pre-employment programs, employment transition programs, apprenticeship initiatives, on-job courses, and certificate programs. E-learning based on Artificial intelligence is beneficial to these programs and skills development in vocational education. A qualitative approach is used on 40 vocational colleges around China to analyze the findings, interviews, and focus group dialogue based on the learning strategies followed by students and teachers. Furthermore, the results of this analysis showed many improvements in the positions of teachers and students in the smart learning program. The study consisted of 102 students, and the results have been checked based on F-Test and TTest

The main contribution of the paper is

- To propose the Advanced Artificial Intelligence assisted Learning model (AAILM) fora smart interactive education system for vocational education.

- Designing the convolutional neural network with Gated Recurrent Units for prediction for students learning performance. 
- The simulation results have been executed, and the suggested AAILM model enhances the student's performance, prediction, engagement, task analysis, efficiency ratio when compared to other existing approaches.

The remainder of the study is organized as follows, section 1 and section 2 deliberated the overview of the smart educational strategies for teachers and students and the existing method. In section 3, Advanced Artificial Intelligence assisted Learning model (AAILM) has been suggested. In section 4 , simulation results have been executed. Finally, section 5 concludes the research article.

\section{Related Works}

Wang et al. [22] suggest the Smart Data Mining Algorithm (SDMA) for smart education. A smart data mining algorithm (SDMA) is utilized to identify and sum up the educational data and enhance teaching information consistency to enhancethe efficiency of the educational system. The algorithm flow and assessment model allows for an optimization and updating framework and develops an appropriate evaluation model through an efficient assessment of the changing state of information preparation. The test findings indicate that the combination of AI and SDMA will increase the teaching and learning performance and strengthen the teaching material that should be further promoted.

Aydoğdu, Ş,et al.[23] discussed the Artificial Neural Network (ANN) for Predicting final student performance in online learning environments.An artificial neural network is shown in e-learning environments to be an important method to forecast student performance. Studies with artificial neural networks typically forecast the student's results, and the use of the learning management system by the students is not concentrated.A prediction of $80,47 \%$ with precision is made of the artificial neural network generated by the study.

Rabiman, $\mathrm{R}$ et al.[24] introduced the Tracking Student Learning And Knowledge (TSLAK) for e-Learning platforms.TSLAK is a model focused on two variables: variables that monitor student learning (VLT) and variables that Track student knowledge (VTK); and models that track students ' knowledge (TSK). This research provides answers about how the model can be developed to track learners' online learning and information on the four e-learning platforms and how VTL and VTK can define and quantify students ' learning and knowledge acquisition processes. Results from VTL and VTK show a significant decrease in the interest of students. 
Grubišić, A et al. [25] suggests the Learning Management System(LMS) for designing and developingthe E-Learning System in vocational education. The goal of Research and development activities is to create a phase-specific LMS-E-learning(LMS-ELS)framework. The LMS is checked by questionnaires and close evaluation by media experts and information experts. This analysis implies that the use of LMS boosts learning success and efficiency.

To overcome these issues, in this paper, Advanced Artificial Intelligence assisted Learning model (AAILM) has been proposed for a smart interactive education system for vocational education. The convolutional neural network with Gated Recurrent Units has been proposed for prediction for students learning performance. The following section 3 discussed the proposed AAILM method briefly.

\section{Advanced Artificial Intelligence assisted Learning model (AAILM)}

In this paper, Advanced Artificial Intelligence assisted Learning model (AAILM) has been proposed for a smart interactive education system for vocational education. Many industries face-demands for skills and the pressure for costs and innovation created by increased globalization. Globalization in certain areas in the responsibility needs extra qualifications and new skills which stay for training which workflows in businesses. In our daily life, digitization and electronic networking are more and more prevalent. The spread of smartphones, computers, and other new devices means that people have access to a growing amount of media. In many enterprises, the need for training then increased. Vocational education and training colleges are supposed to provide students with the skills needed by the businesses that lead to the most modern technology to fulfill these that needs of industries. However, this improved demand for training can no longer be met solely by the teaching space curriculum due to the available resources and cost pressures. Electronic learning has been supportive of the appearance of new digital media. Consequently, the utilization of electronic media plays a significant role in vocational colleges. New forms of learning and teaching are increasingly important in vocational colleges under the term "e-learning."

The convolutional neural network with Gated Recurrent Units has been proposed for prediction for students learning performance. Artificial intelligence supported e-learning platform are extensively utilized by vocational educational institutions to guarantee better teaching and learning experiences along with career enhancements. In China, vocational education and training apply to train and professional qualifications offered by various programs such as pre-employment programs, employment transition programs, 
apprenticeship initiatives, on-job courses, and certificate programs. E-learning based on Artificial intelligence is beneficial to these programs and skills development in vocational education.

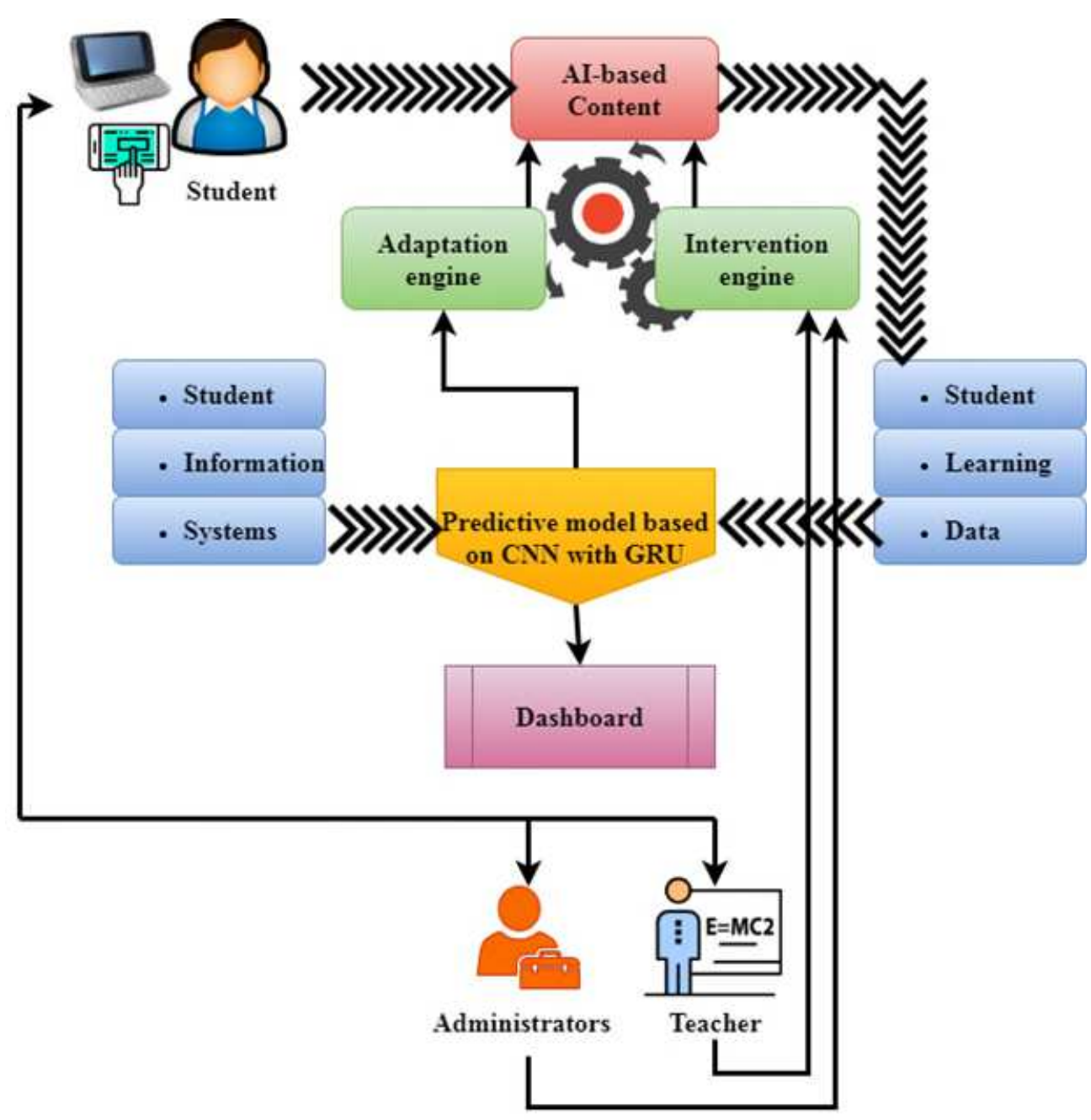

Figure 1: The architecture of the proposed Advanced Artificial Intelligence assisted Learning model (AAILM)

Figure 1 shows the architecture of the proposed advanced artificial intelligence assisted learning model (AAILM).Formal education offers classes or seminars for which formal registration is required. These activities are planned, planned, and held at specified times by a course provider. A fee is usually expected, and the learning process should be organized by a virtual lecturer/teacher/contact person. In comparison, formal electronic learning is related to learning goals and skills and ends with assessment, qualification, or authorization. The learning process takes place on an informal e-learning basis, in a more or less unplanned way. There is no teacher, and the learner must discover the information individually.The learner attempts to discover the requisite knowledge (learning by doing) independently. Only when a 
teacher designs and plans the education in a teaching and technique way is the deliberate elearning. Therefore, a teacher-coordinated or manageable idea is an "intended," learning concept.The learning content based on multimedia presentation through visualization, animations, simulation, playful learning situations, and interactive and realistic exercises enhances the learning progression and encourages learners. The access and use of connectivity and collaboration scenarios in virtual teaching space among teachers and learners and in groups of students are additional benefits (search tools, links, online libraries, glossaries, etc.). Teachers are not anxious that before most participants, they have their knowledge gaps uncovered. Further monetary savings are made by removing the cost of travel and accommodation.

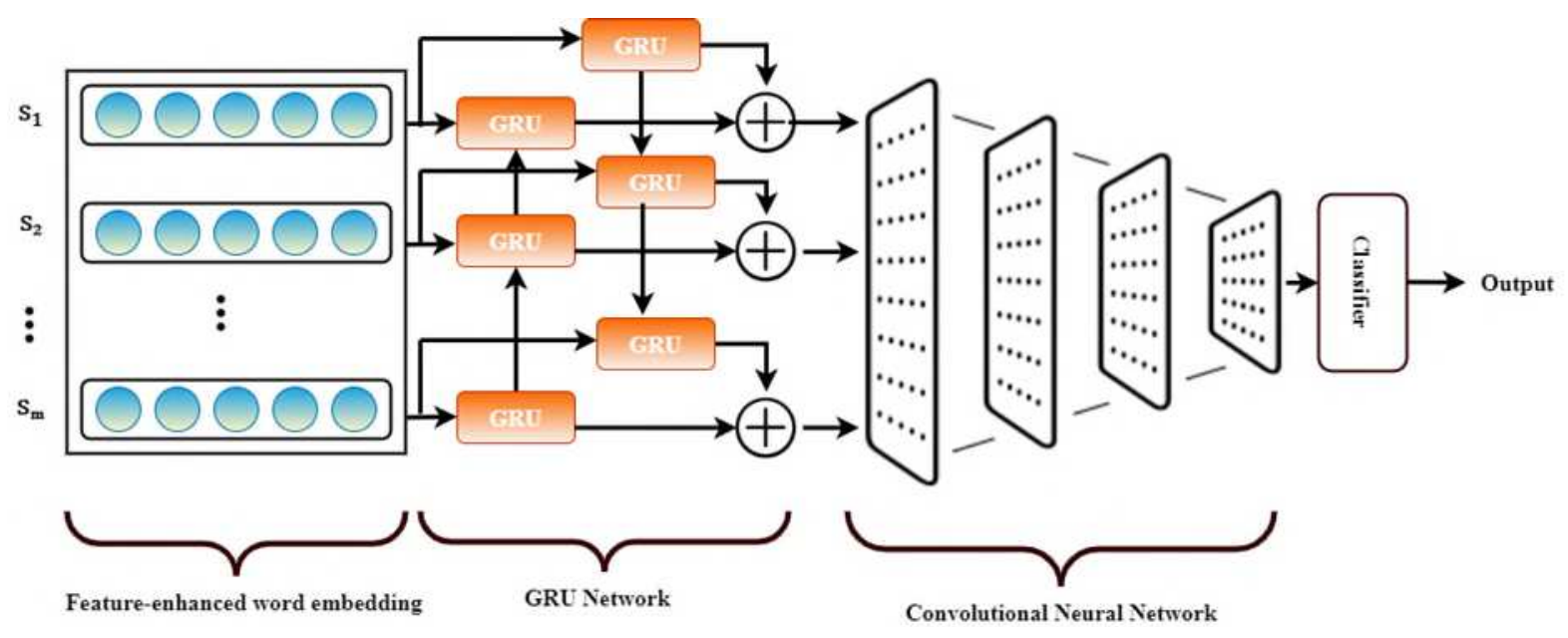

\section{Figure 2: CNN with GRU network}

Figure 2 shows the CNN with GRU network. Analysis of the large volumes of multi-modal data is a problem in e-learning environments. The mainprerequisite for creating an effective prediction model is to identify a reliable fault prediction model. Raw data is mostly semistructural or structured in various ways in the e-learning framework, such as documents, photos, files, logs, etc. Such data is difficult to analyze directly and must be translated into a machine /output format, and quantification of features is one of the most commonly applied methods.GRU is a sort of recurrent neural network. LSTM,based on the RNN variants, can be utilized to collect the sequence data 's long dependency features. The configuration of the LSTM is relatively complicated, though, making the training and prediction of the LSTM much longer. The main variances between a Gated recurrent unit cell and a Long short term memory are that a Gated recurrent unit cell has two gates (update and reset gates) in place of three (output, input, and forget gate) further discovery of the LSTM. With its 
superior gate-structure, Gated recurrent unit cellmight achieve asimilar effect as long shortterm memory on the condition that the model has less parameters and ease convergence, which is appropriate for fast learning prediction.

Statement 1: Let's consider a set of lessons in an online course $C^{K}=\left\{k_{1}, k_{2}, \ldots k_{j}, \ldots k_{n}\right\}$ and set of students $C^{W}=\left\{w_{1}, w_{2}, \ldots w_{i} \ldots w_{m}\right\}$, where $k_{j}$ and $w_{i}$ indicates the $j$ th lesson and $i t h$ student, correspondingly. The features from $w_{i}$ in the lesson $k_{j}$ indicated by $U_{j i}=$ $\left\{u_{j i 1}, u_{j i 2}, \ldots u_{j i c}\right\}$. The last performance of the student is represented by $K_{i}$. In addition, in every lesson, data has been collected as follows:

- The basic information (age, ID, target learning, gender) of every user is indicated by $w_{i}\left(I D_{i}, A g e_{i}, G_{i}, T_{j}\right)$

- The information keywords set of every lesson is represented by $L_{j}$

- The qth activity (contents, behaviors,) and its logs record (begin time, location, completionperiod, device) of $w_{i}$ in $k_{j}$ are indicated by $B_{j i q}\left(A_{j i q}, D_{j i q}\right)$ and $\operatorname{logs}\left(r_{j i q}, r_{j i q}^{\prime}, \operatorname{Logs}_{j i q}, C_{j i q}\right)$

- The homework mark of $w_{i}$ in $k_{j}$ is $H_{j i}$.

Statement 2: Feature quantification of learning. For $w_{i}$ in $k_{j}$, engagement $\mathbb{E}_{j i}$, the relevance of contents $\mathbb{C}_{j i}$, liveness $\mathbb{A}_{j i}$, time $\mathbb{T}_{j i}$, sentiments $\mathbb{S}_{j i}$, target matching rate $\mathbb{M}_{j i}$, homework $\mathbb{H}_{j i}$ has been represented. For $w_{i}$ in $k_{j}$,

$\mathbb{E}_{j i}=\sum_{q=1}^{Q} E\left(\operatorname{Logs}_{j i q}\right), E\left(\operatorname{Logs}_{j i q}\right)=\left\{\begin{array}{rr}1, & r_{j i q}^{\prime}-r_{j i q} \geq \Phi \\ 0, & 0<r_{j i q}^{\prime}-r_{j i q}<\Phi\end{array}\right.$

As inferred from the equation (1), where $\Phi$ denotes the time threshold.

$\mathbb{A}_{j i}=\sum_{q=1}^{Q} A_{j i q} A_{j i}=\left\{\alpha_{1}, \alpha_{2}, \alpha_{3}, \alpha_{4}, \alpha_{5}, \alpha_{6}\right\} \in[0,1]$

As shown in equation (2) where $\left\{\alpha_{1}, \alpha_{2}, \alpha_{3}, \alpha_{4}, \alpha_{5}, \alpha_{6}\right\}$ denotes the posting, browsing, searching, commenting, collecting, and praising, correspondingly. According to the features of datasets in this study, the respective value $\{0.1,0.6,0.7,0.3,0.5,0.6\}$.

$\mathbb{C}_{j i}=\operatorname{sim}\left(L_{j}, H_{j i}\right)$ 
As derived in equation (3) where $H_{j i}$ is word set which is created from a top $-l$ word count evaluating of the entire interactive contents for $w_{i}$ in $k_{j} . \operatorname{sim}\left(L_{1}, L_{2}\right)$ denotes the semantic similarity between a two-word set $L_{1}$ and $L_{2}$. In specific, $l_{1}^{(b)} \in L_{1}$ and $l_{2}^{(a)} \in L_{2}$,

$C\left(l_{1}, l_{2}\right)=\min \left(\operatorname{edge}\left(l_{1}, l_{2}\right)\right)$

$\operatorname{sim}\left(L_{1}, L_{2}\right)=\sum_{l_{1}^{(b)}}^{L_{1}} \sum_{l_{2}^{(a)}}^{L_{2}} \frac{\beta \times\left(\mathcal{K}_{1}^{(b)}+\mathcal{K}_{2}^{(a)}\right)}{\left(c\left(l_{1}^{(b)}, l_{2}^{(a)}\right)+\beta\right)+\max \left(\left|\mathcal{K}_{1}^{(b)}+\mathcal{K}_{2}^{(a)}\right|, 1\right)}$

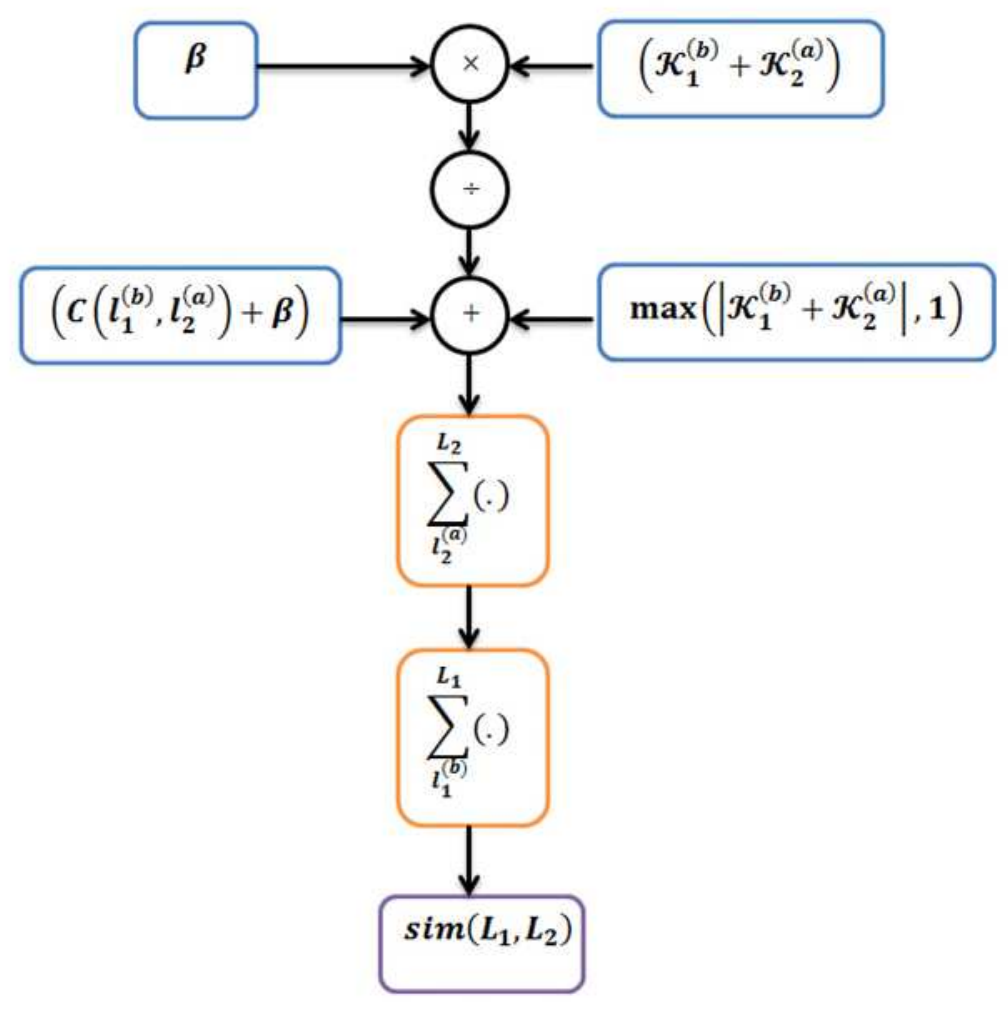

\section{Figure 3: Similarity index}

As inferred from the equation (4), (5) and figure 3 shows the similarity index, where $C\left(l_{1}, l_{2}\right)$ indicates the semantic distance between words $l_{1}$ and $l_{2} \cdot \mathcal{K}$ denotes the semantic hierarchy, and $\beta(\beta>0)$ indicates a control variable.

$\mathbb{S}_{j i}=\delta_{1} P\left(D_{j l}\right)+\delta_{2} F D_{j i}$

As discussed in equation (6) where $P\left(D_{j l}\right)$ is a function of optimization to determine the text contents sent by $w_{i}$ in $k_{j}$, which gives an outcome in the range of $[1,-1]$, to identify the sentiment state of $w_{i}$. Besides, $F D_{j i}$ is a measurement of various sentiments, with otheroutcome range of $[1,-1]$. Further, $\delta_{1}, \delta_{2}$ are two weighted attributes. 


$$
\begin{aligned}
& \mathbb{T}_{j i}=\sum_{q=1}^{Q}\left(r_{j i q}^{\prime}-r_{j i q}\right) \\
& \mathbb{M}_{j i}=\sum_{o=1}^{O} \operatorname{sim}\left(L_{j}, J S_{i o}\right) *\left(1-\tanh \left(\frac{o-1}{5}\right)\right)
\end{aligned}
$$

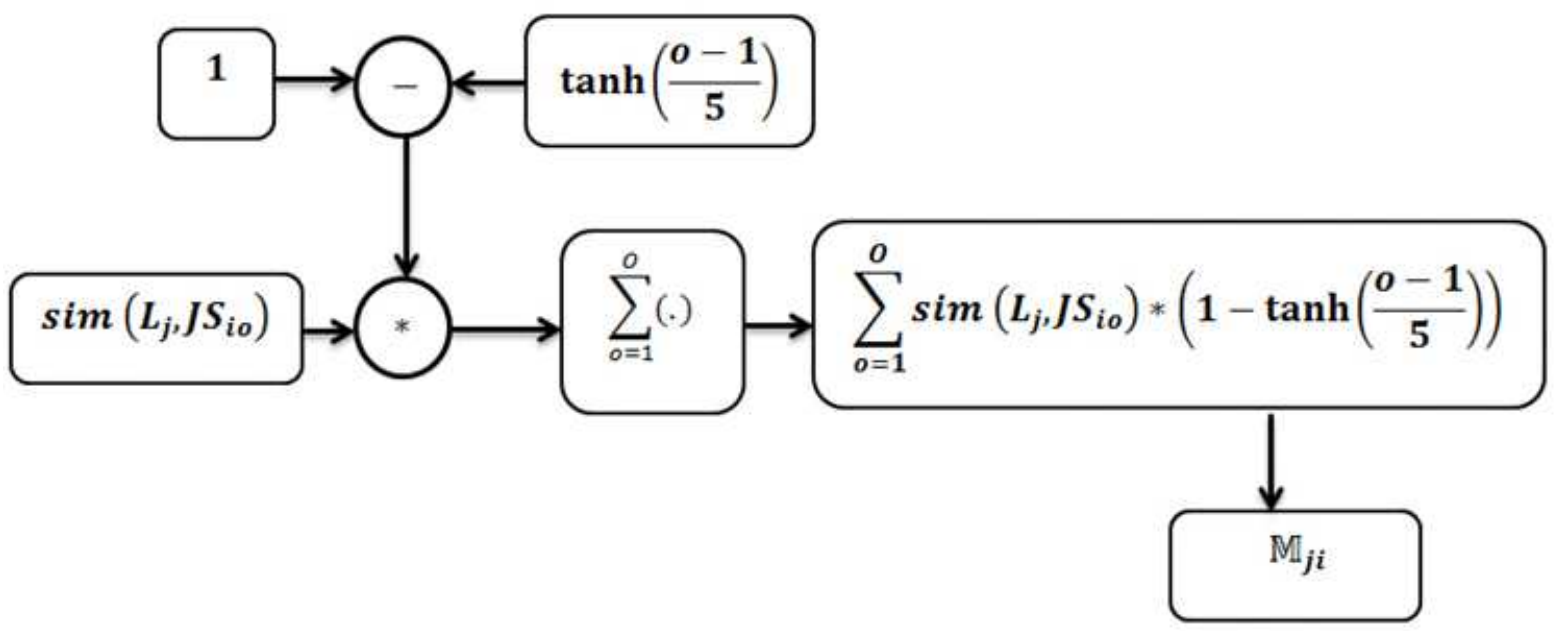

\section{Figure 4: Learning Targets}

As acquired from equation (7), (8) and figure 4 shows the learning targets, where $S$ denotes the initial learning targets words with $O$ words, $1-\tanh \left(\frac{o-1}{5}\right)$ provides weighted attributes for various words.

Moreover, the learning features comprise some fundamental information, that is ID number $I D_{i}$, age $A g e_{i}$, gender $G_{i}$, and the type of device $C_{i}$.

$U_{i} \subseteq\left\{\mathbb{E}_{j i} \cup \mathbb{A}_{j i} \cup \mathbb{C}_{j i} \cup \mathbb{S}_{j i} \cup \mathbb{T}_{j i} \cup \mathbb{M}_{j i} \cup \mathbb{H}_{j i} \cup G_{i} \cup A g e_{i} \cup C_{i}\right\}$

Based on this equation (9), students' task analysis and decision-making skills have been identified.

Statement 3:K denotes the student learning performance in the final exam, that involves 3 levels (Very Good, Good, Medium, Poor). In education, $\rho$ and $\mu$ are the number of studentsstandard deviation and mean of the samples to guarantee the information balance of every class. $w_{i}$ in this course, feature matrix input $U_{i} \in \mathbb{R}^{c \times n}$, and its output is $K_{i} \in$ $\{A, B, C, D\}$. Based on these assumptions, a student's performance has been analyzed.

Statement 4: The prediction model, which creates the input feature into a vector of variablelength, i.e., in the $j t h$ lesson of the $\operatorname{progression}(j<n)$, the $1^{\text {st }}$ rows of the matrix $U_{i} \in$ 
$\mathbb{R}^{c \times n}$ have information, while the latter is empty. Therefore,the filling of missing data is important to ensure the wholematrix structure. Thus, reviewing the features of education, the empty data has been filled with a weighted-average based on the present state. Assumed a threshold $\varepsilon$ means a minimum constraint of lessons, eliminating any nominal in the dataset in which thelesson course length is lesser than $l$. Supposing the number of the present lesson is $d$, the weight dynamic $\omega_{d}$ is provided as

$\omega_{d}=\frac{\ln d+1}{\sum_{j=1}^{n}(\ln j+1)}$

Statement 5: Convolutional neural network has been successfully utilized to recognize tasks. Feature extraction is constructed to depict the efficientportion with quick processing. With numerousextraction of feature phases in grading stacking, this systemcontainsnon-linear transformation layers, subsampling layers, pooling layers, and filter layers.

The input layerdepends on the preassumption and characterization, mlearning student in this course, and every student gets a matrix $U_{i}$ of size $c \times n$, and the input $3 \mathrm{D}$ tensor $U \in$ $\mathbb{R}^{c \times n \times m}$, The featureslearning in the matrix all are independent, and lessons are connecteddue to the knowledge association between lessons.

Using non-linearity and a set of convolution filters, the filter layer collects the data. Around every feature in $U_{i}$, this layer can be realized as $L$ sets of $c \times t(t<n) 2 \mathrm{D}$ filter $D_{i}$ at every stage. Over the convolution through input $Y$ and kernels $D$,

$y_{i}^{k}=f\left(\sum_{j=1} l_{j i} * y_{j}^{k-1}+a_{i}\right)$

As shown in equation (11) where the $y_{i k}$ is the output in $k t h$ feature map input element in the $(k-1)$ th and feature map is indicated $y_{j}^{(k-1)} \cdot l_{k j i}$ and $a_{k i}$ are the trainable variables in the convolutional operation.

Subsampling and average pooling layer for student learning prediction tasks should consider more attention rather than image feature. Though in certain situations, max-pooling generally has good performance,the distribution of the extracted features can impact. Average pooling is better suited to this analysis. To alleviate the issue of feature vanishing impacted by an inadequate number of lessons, the threshold $\xi$, if $n>\xi$, by anID pooling size of the pool $c w$, output obsessed by the size $c \times(n / c w)$ is generated. Every feature map output is then sub- 
sampled by a factor $c w$ vertically; these matrix dimensions can be evaluated as the feature extracted.

Statement 6: Gated recurrent unit cell (GRU) is a memory cell consisting of 4keyelements, update gate, reset gate, proposed unit, and candidate hidden state. Update andReset gates provide cells to access data for a long intervaland provides memory. The long-temporal dependences learning block proposed unit computes the hidden conditions by a set of expressions.

$r_{t}=\rho\left(S_{r} \cdot\left[g_{t-1}, y_{t}\right]\right)$

$g_{t}=\left(1-z_{t}\right) * g_{t-1}+z_{t} * \tilde{g}_{t}$

As inferred from the equation (12) where $r_{t}, \square_{\square}$ indicates the reset gate and update gate, $\widetilde{\square}_{\square}$ indicates the candidate hidden layer and $\square_{\square}$ is the Gated recurrent unit cell output.

The proposed Advanced Artificial Intelligence assisted. Learning model (AAILM) for a smart interactive education system for vocational education achieves high student performance, prediction, engagement, task analysis, efficiency ratio when compared to other existing methods.

\section{Results \& Discussion}

The proposed Advanced Artificial Intelligence assisted. Learning model (AAILM) experimental results have been performed. A qualitative approach is used on 40 vocational colleges around China to analyze the findings, interviews, and focus group dialogue based on the learning strategies followed by students and teachers. Furthermore, the results of this analysis showed many improvements in the positions of teachers and students in the smart learning program.The study consisted of 102 students, and the results have been checked based on F-Test and T-Test. The result findings revealed that the academic successes of students in traditional and vocational colleges vary significantly and computed with graphical illustration as well as case study. The use of smart instruction has dramatically increased the success of teachers and students has been firmly researched in this paper.

\section{i) Prediction Ratio Analysis}


The vocational students learning is improved by applying the e-learningconcept, and the prediction of student's performance is most important for vocational education. The main aim of this smart education based onthe Advanced Artificial Intelligence assisted Learning model AAILM is to prove the possibility of training for vocational students' education. The feasibility of creating a learning and to enhancethe capability of students in vocational education based on AI techniques. Figure 5demonstrates the prediction ratio. The experimental result shows the AAILM and e-learning to better accurate prediction is achieved by analyzing student learning when compared to other methods.
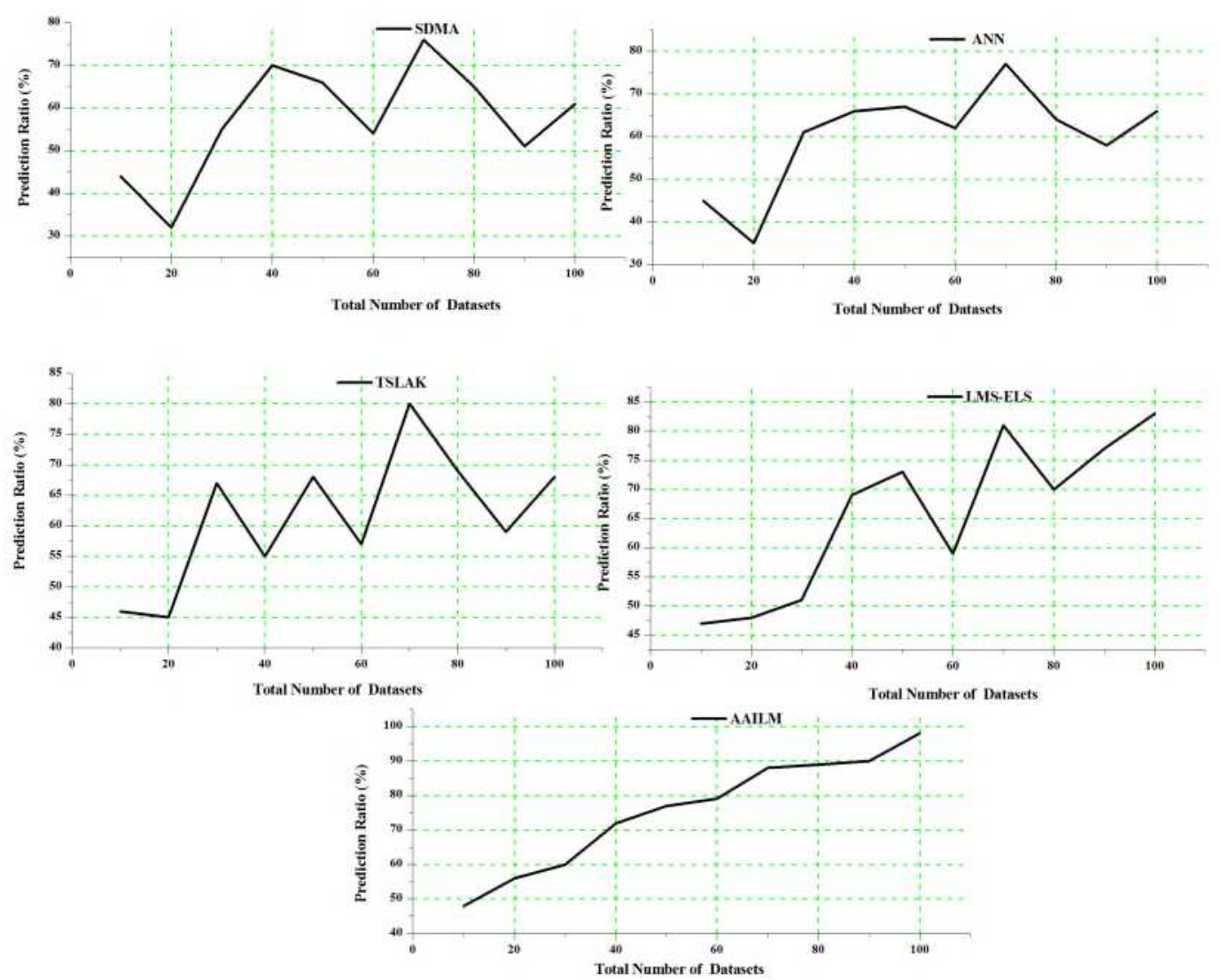

\section{Figure 5: Prediction Ratio}

\section{ii) Student performance Ratio:}

This paper presents the Advanced Artificial Intelligence assisted Learning model (AAILM), which is used to improvethe teaching and learning of vocational students.This AAILM provides e-learning with education to better understanding the study for students. This improves the student performance in education and its relief the student carrier stress. The performance of the e-learning is well improved and the knowledge of vocational students 
to concentrate the carrier to achieve their goal based ondecision making skill. Figure 6 shows the student performance ratio. The results of AAILM with e-learning in vocational education offersa student to enhance the performance in academic and their carrier when compared to other methods.
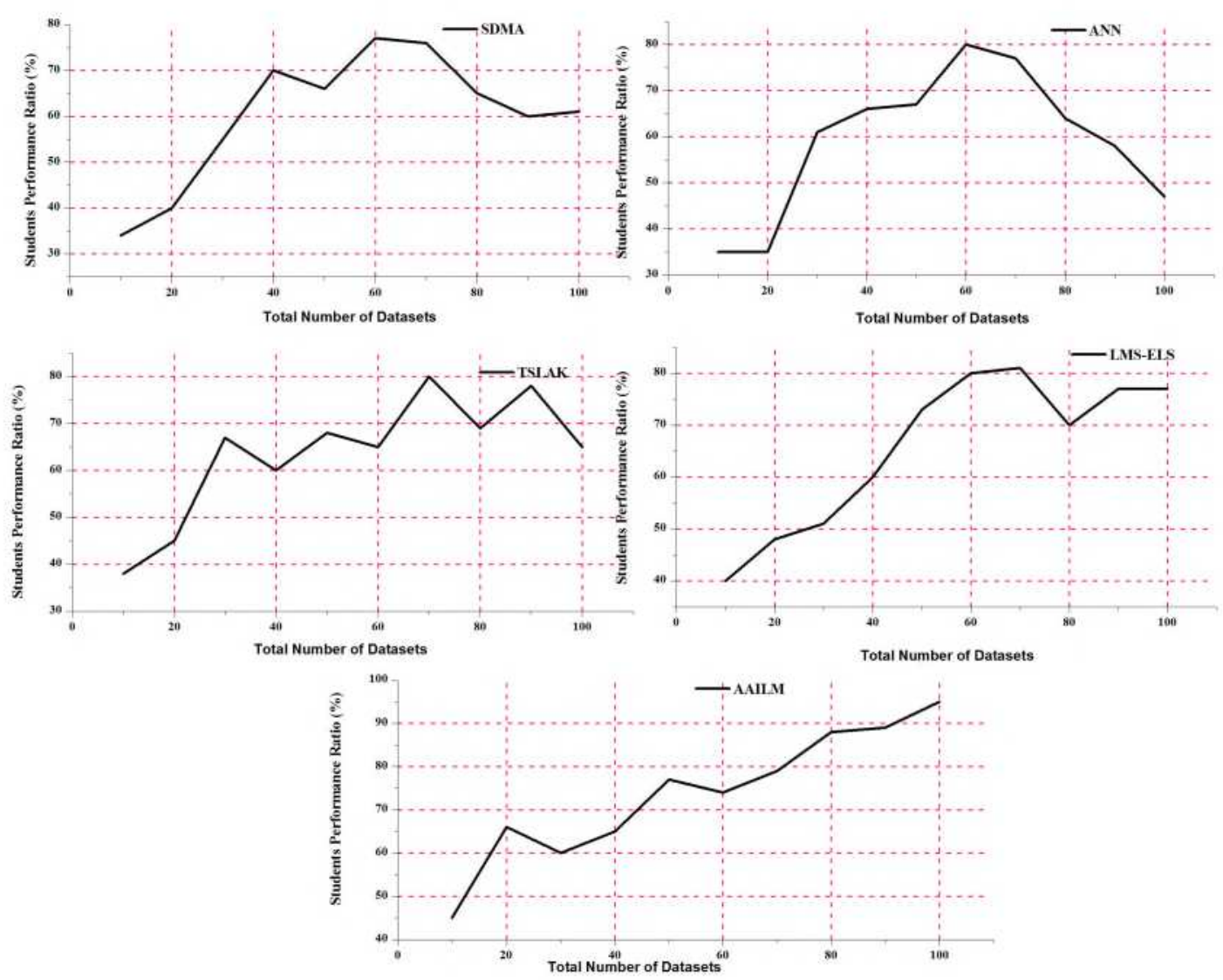

\section{Figure 6: Students Performance Ratio}

\section{iii) Efficiency Ratio}

The proposed Advanced Artificial Intelligence assisted Learning model (AAILM) is used to design a smart interactive education system for a vocational college. E-learning based on AAILM help students to be smarter, more effective, more relaxed, and flexible. Learners use intelligent equipment to access digital services across the Internet and immerse themselves in specialized and seamless learning. Intelligent education has received expanded popularity, a term that defines new-age learning. Figure 7 shows the efficiency ratio. The experimental results AAILM improvedthe efficiency of learning for students compared to other methods. 

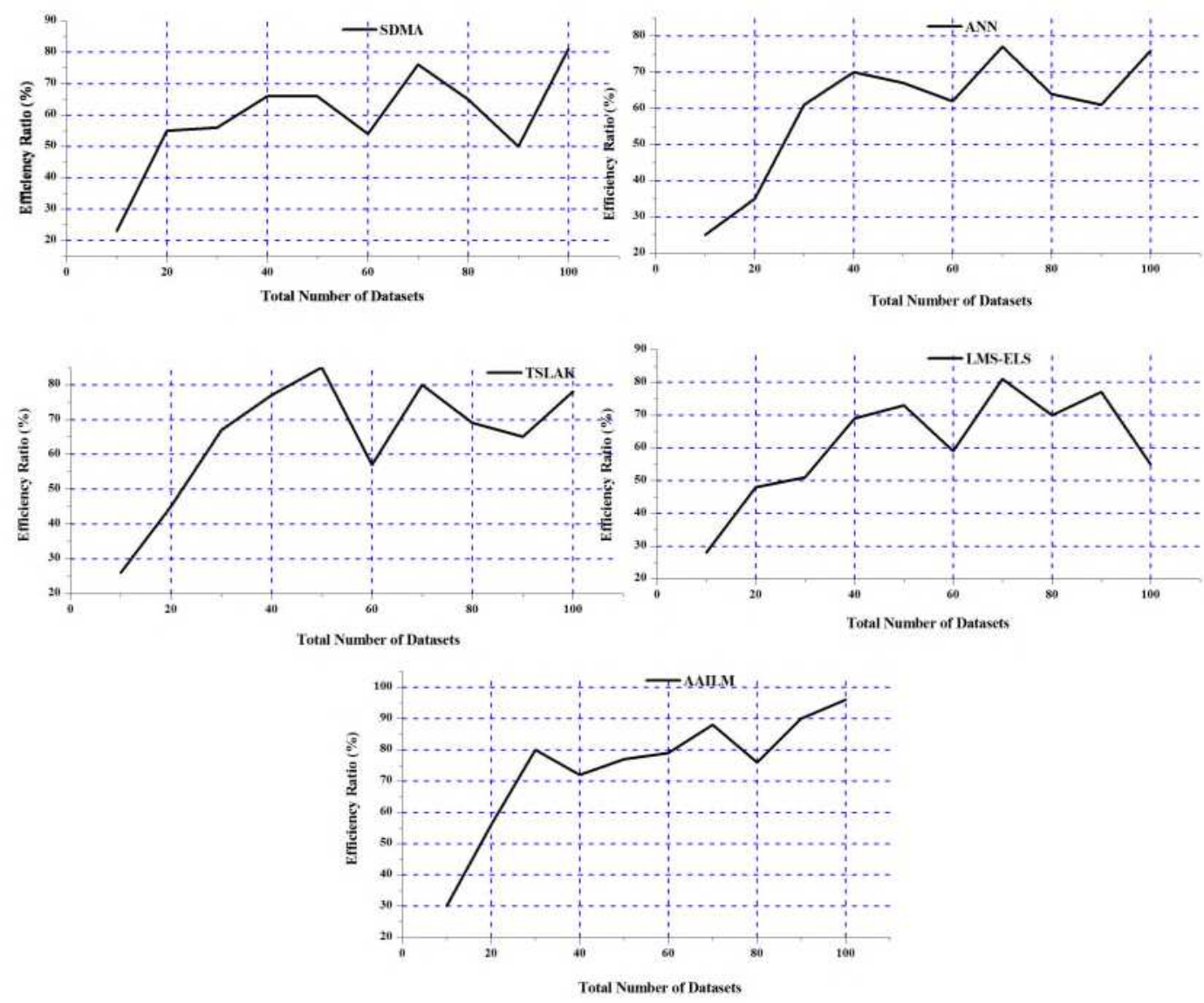

\section{Figure 7: Efficiency Ratio}

\section{iv) Student task analysis Ratio}

The proposed Advanced Artificial Intelligence assisted Learning model (AAILM) is used to create a smart digital educational system for vocational colleges. Artificial intelligence has been used mostly in several tasks to build expertise and training methods for vocational education. AI will help fill needs in the gap in studying and teaching and encourage vocational college teachers to achieve better than traditional strategies. The artificial intelligence is creating students and teacher interactions for better decision making to complete the task.Figure 8 shows the Student task analysis. The experimental results show the student decision making to fill their educational needs compared with other methods. 

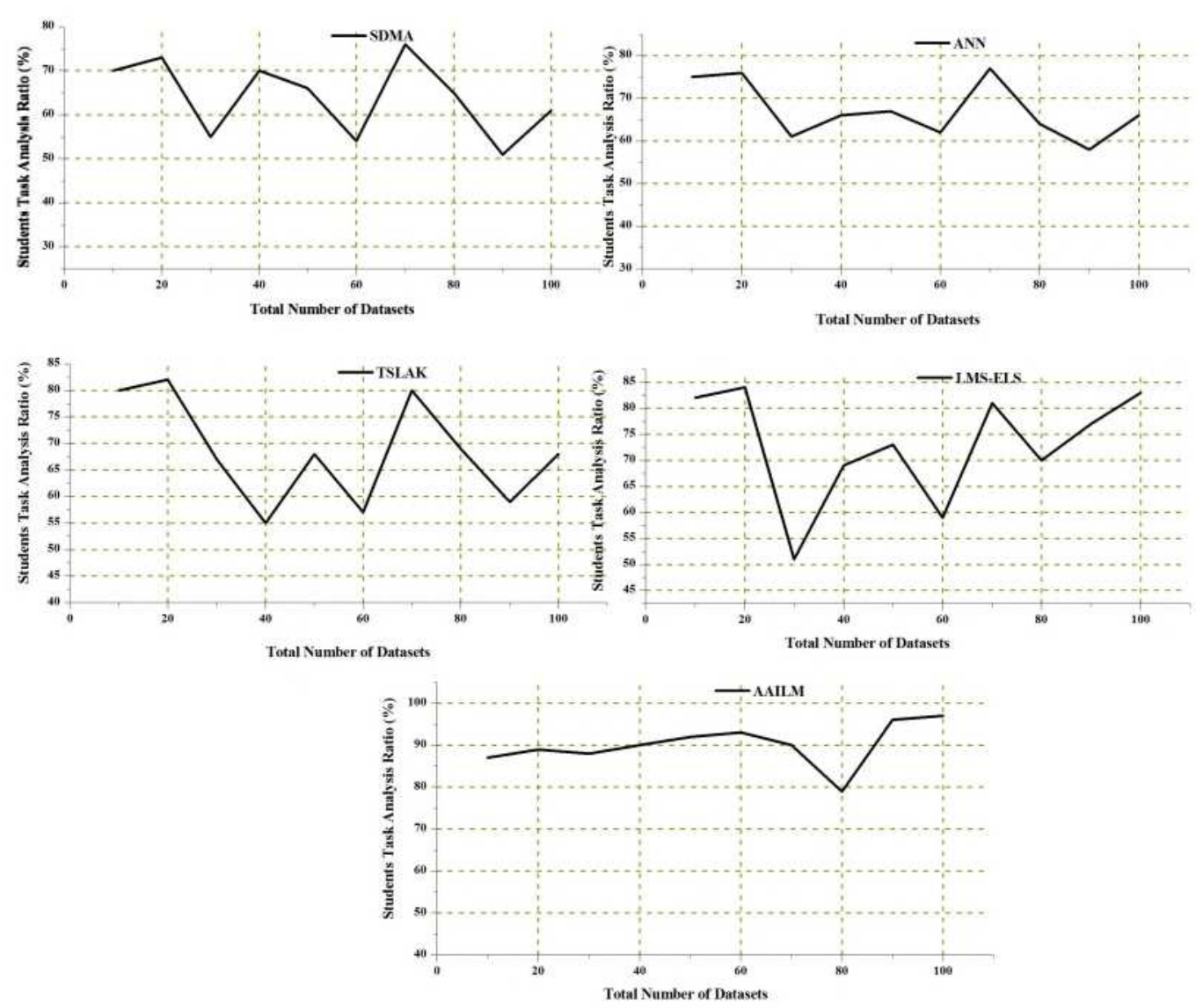

\section{Figure 8: Students Tasks Analysis Ratio}

\section{v) Engagement Ratio:}

Vocational colleges use Advanced Artificial Intelligence assisted Learning model AAILM to help students achieve their success. The study consisted of 102 students, and the results have been checked based on F-Test and T-Test. The result findings revealed that the academic successes of students in traditional and vocational colleges vary significantly and computed with graphical illustration as well as case study.These AAILM models can effectively be implemented into vocation education instruction to help teachers assess students' involvement in learning concerning various tasks and resources and offer additional interventions for students before their final test. This study examined the relationship between the engagement of students and the evaluation score of courses. Table 1 shows the engagement ratio. The experimental results of student engagement in their education improve student learning when compared to other methods. 
Table 1: Engagement ratio

\begin{tabular}{|l|l|l|l|l|l|}
\hline $\begin{array}{l}\text { Total } \\
\text { Number of } \\
\text { Datasets }\end{array}$ & SDMA & ANN & TSLAK & LAM-ELS & AAILM \\
\hline 10 & 65.5 & 70.2 & 71.4 & 73.1 & \\
\hline 20 & 65.3 & 64.3 & 63.2 & 65.2 & 75.5 \\
\hline 30 & 51.2 & 54.6 & 56.2 & 57.3 & 59.2 \\
\hline 40 & 46.4 & 47.4 & 48.1 & 49.4 & 55.2 \\
\hline 50 & 32.4 & 43.1 & 54.3 & 65.5 & 77.3 \\
\hline 60 & 43.6 & 55.2 & 56.5 & 63.6 & 61.3 \\
\hline 70 & 73.7 & 77.3 & 78.5 & 71.7 & 80.4 \\
\hline 80 & 83.8 & 82.2 & 86.3 & 84.7 & 89.6 \\
\hline 90 & 76.7 & 74.3 & 72.4 & 70.8 & 92.7 \\
\hline 100 & 87.9 & 89.5 & 75.5 & 71.9 & 94.6 \\
\hline
\end{tabular}

The proposed Advanced Artificial Intelligence assisted. Learning model (AAILM) for a smart interactive education system for vocational education achieves high student performance, prediction, engagement, task analysis, efficiency ratio when compared to other existing Smart Data Mining Algorithm (SDMA), Artificial Neural Network (ANN), Tracking Student Learning And Knowledge (TSLAK), Learning Management System based Elearning framework (LMS-ELS) methods.

\section{Conclusion}

This paper presents the Advanced Artificial Intelligence assisted Learning model (AAILM) for a smart interactive education system for vocational education. The convolutional neural network with Gated Recurrent Units has been proposed for prediction for students learning performance.Compared to conventional classroom teaching, e-learning based on artificial intelligence has advantages. This paper looked at the benefits and drawbacks from two perspectives: the point of view of the learner (apprentices, staff, teachers, trainees) and the supplier (VET institution and training center). In the view of the instructor or instructor, the discussion of the advantages and advantages was deliberately omitted as it is overlapping. Students value eLearning as flexibility in selecting the right location, time, and learning pace, minimizing learning time through the individualization of the learning content, increasing 
incentive to learn through multimedia presentations, getting access to accessible libraries, and using interactive classrooms; and removing travel and accommodation costs. E-learning based on the AI method enhances the employment rate witha students' intelligence and critical-thinking skills and prepares a student for a particular job.The simulation results show that the suggested AAILM model enhances the student's performance ratio of $98.6 \%$, prediction ratio of $95.7 \%$, student engagement ratio of $94.6 \%$, task analysis ratio of $96.8 \%$, an efficiency ratio of $97.8 \%$ when compared to other existing methods.

Ethics Declarations

Conflict of interest

The authors declare that they have no conflict of interest.

Ethical approval

This article does not contain any studies with human participants or animals performed by any of the authors.

Author Statement

Conception and design of study : XiaobingYu

Acquisition of data : XiaobingYu

Analysis and/or interpretation of data : XiaobingYu

\section{Reference:}

1. Hoerunnisa, A., Suryani, N., \& Efendi, A. (2019). The Effectiveness of the Use of Elearning in Multimedia Classes to Improve Vocational Students' Learning Achievement and Motivation. Kwangsan, 7(2), 295731.

2. Mehta, A., Morris, N. P., Swinnerton, B., \& Homer, M. (2019). The influence of values on E-learning adoption. Computers \& Education, 141, 103617.

3. STAPA, M. A., \& MOHAMMAD, N. (2019). THE USE OF ADDIE MODEL FOR DESIGNING BLENDED LEARNING APPLICATION AT VOCATIONAL COLLEGES IN MALAYSIA. Asia-Pacific J. Inf. Technol. Multimed, 8(1), 49-62. 
4. Gao, J., Wang, H., \& Shen, H. (2020, May). Smartly handling renewable energy instability in supporting a cloud datacenter. In 2020 IEEE international parallel and distributed processing symposium (IPDPS) (pp. 769-778). IEEE.

5. Dovbenko, S., Naida, R. G., Beschastnyy, V. M., Bezverkhnia, H. V., \&Tsybulska, V. V. (2020). Problem of Resistance to the Introduction of Distance Learning Models of Training in the Vocational Training of Educators. International Journal of Learning, Teaching, and Educational Research, 19(2), 1-12.

6. Gheisari, M., Najafabadi, H. E., Alzubi, J. A., Gao, J., Wang, G., Abbasi, A. A., \& Castiglione, A. (2021). OBPP: An ontology-based framework for privacy-preserving in IoT-based smart city. Future Generation Computer Systems, 123, 1-13.

7. Wicaksono, A., Florentinus, T. S., \& Ahmadi, F. (2020). Development of E-Learning in Web Programming Subjects for Moodle Based Vocational Students. Innovative Journal of Curriculum and Educational Technology, 9(1), 1-9.

8. Manogaran, G., Thota, C., Lopez, D., Vijayakumar, V., Abbas, K.M., Sundarsekar, R.: Big Data Knowledge System in Healthcare. InInternet of Things and Big Data Technologies for Next Generation Healthcare, pp. 133-157. Springer, Berlin (2017)

9. Sarwandi, S., Giatman, M., Sukardi, S., \& Irfan, D. (2019). Developing mobile-based project-based learning module for project management courses in vocational education. Jurnal Pendidikan Vokasi, 9(2), 207-216.

10. Agustini, K., \&Tegeh, I. M. (2019). Learning Style Tendency Analysis for Vocational Students. JPhCS, 1165(1), 012022.

11. Tharwat, A., Mahdi, H., Elhoseny, M., Hassanien, A.E., Recognizing human activity in mobile crowd sensing environment using optimized k-NN algorithm, Expert Systems with Applications 107, pp. 32-44, 2018

12. Arifin, Z., Nurtanto, M., Priatna, A., Kholifah, N., \&Fawaid, M. (2020). Technology Andragogy Work Content Knowledge Model as a New Framework in Vocational Education: Revised Technology Pedagogy Content Knowledge Model. Online Submission, 9(2), 786-791.

13. Shakeel, P. M., \& Baskar, S. (2020). Automatic Human Emotion Classification in Web Document Using Fuzzy Inference System (FIS): Human Emotion Classification. International Journal of Technology and Human Interaction (IJTHI), 16(1), 94-104. DOI: 10.4018/IJTHI.2020010107

14. Yağci, A., \&Cevik, M. (2019). Prediction of academic achievements of vocational and technical high school (VTS) students in science courses through artificial neural 
networks (comparison of Turkey and Malaysia). Education and Information Technologies, 24(5), 2741-2761.

15. Cox, D., \&Prestridge, S. (2020). Understanding fully online teaching in vocational education. Research and Practice in Technology Enhanced Learning, 15(1), 1-22.

16. Petrenko, L., Kravets, S., Bazeliuk, O., Maiboroda, L., \&Muzyka, I. (2020). Analysis of the current state of distance learning in the vocational education and training institutions. In E3S Web of Conferences (Vol. 166, p. 10010). EDP Sciences.

17. Bochkareva, T. N., Akhmetshin, E. M., Zekiy, A. O., Moiseev, A. V., Belomestnova, M. E., Savelyeva, I. A., \&Aleynikova, O. S. (2020). The Analysis of Using Active Learning Technology in Institutions of Secondary Vocational Education. International Journal of Instruction, 13(3), 371-386.

18. Warju, W., Ariyanto, S. R., Soeryanto, S., Hidayatullah, R. S., \&Nurtanto, M. (2020). Practical Learning Innovation: Real Condition Video-Based Direct Instruction Model in Vocational Education. Journal of Educational Science and Technology (EST), 6(1), 79-91.

19. Radosavljevic, S., Radosavljevic, V., \&Grgurovic, B. (2020). The potential of implementing augmented reality into vocational higher education through mobile learning. Interactive Learning Environments, 28(4), 404-418.

20. SenelTekin, P., Ilgaz, H., AfacanAdanir, G., Yildirim, D., \&Gülbahar, Y. (2020). Flipping e-Learning for Teaching Medical Terminology: A Study of Learners' Online Experiences and Perceptions. Online Learning, 24(2), 76-93.

21. ÖZKAN, U. B., Cigdem, H., \& Erdogan, T. (2020). ARTIFICIAL NEURAL NETWORK APPROACH TO PREDICT LMS ACCEPTANCE OF VOCATIONAL SCHOOL STUDENTS. Turkish Online Journal of Distance Education, 21(3), 156169.

22. Wang, S. (2019). Smart data mining algorithm for intelligent education. Journal of Intelligent \& Fuzzy Systems, 37(1), 9-16.

23. Aydoğdu, Ş. (2020). Predicting student final performance using artificial neural networks in online learning environments. Education and Information Technologies, 25(3), 1913-1927.

24. Rabiman, R., Nurtanto, M., \&Kholifah, N. (2020). Design and Development ELearning System by Learning Management System (LMS) in Vocational Education. Online Submission, 9(1), 1059-1063 
25. Grubišić, A., Žitko, B., Stankov, S., Šarić-Grgić, I., Gašpar, A., Tomaš, S., ... \&Dodaj, A. (2020). A common model for tracking student learning and knowledge acquisition in different e-Learning platforms. Journal of e-Learning and Knowledge Society, 16(3), 10-23. 